\title{
Research on the physical fatigue recovery and Training Method of Taekwondo Athletes
}

\author{
Qi Lv \\ Wuhan Technology and Business University, Wuhan, China
}

Keywords: Taekwondo Athletes; Training Method; fatigue recovery.

\begin{abstract}
Through the origin of fatigue recovery training, fatigue recovery competitive ability structure, fatigue recovery physical training content, fatigue recovery training and traditional training aspects such as comparative analysis the content of study provide theoretical basis for fatigue recovery the taekwondo training, then according to the design principles and fatigue recovery training according to fatigue recovery fitness training taekwondo project characteristics .By combining the characteristics of Taekwondo project and daily physical training, mainly in anaerobic glycolysis for energy training, core strength training, agility training, speed-strength and lower limb knee strength training.
\end{abstract}

\section{Introduction}

Taekwondo is an old and young sports, which is a form of the martial arts, with the development and popularization of taekwondo as well as the international sports exchange of boxing, it gradually developed into a formal event. Taekwondo belongs to the item of competition project in the same field, which is a competition with strength, speed, stamina, skill and intelligence. Therefore, the level of athlete's physical ability has increasingly become the key to ensure the large load exercise intensity for the competitive games in multi period. In order to make the athletes achieve good sports performance, in addition to techniques and tactical training, the fatigue recovery problem of taekwondo athletes' has received more and more attention. Physical fitness is the foundation of taekwondo movement, and physical deficiencies in Taekwondo will severely restrict technical and tactical level of play. With the improvement of taekwondo competition level, the change of the rules, as well as more attention and investment from the countries, competitions in taekwondo which put forward higher requirements on the Taekwondo athlete's fitness level , are facing increasingly fierce antagonism. Although the research in Taekwondo physical monitoring and training methods is still in weak phase, In training practice, the general coach attaches great importance to taekwondo player's physical ability training.

\section{The Concept of Sports Fatigue}

According to Sports Physiology, the definition of sports fatigue is: the phenomenon that exercise capacity and body function is temporarily declined caused by the sports exercise. In 1982, in the Fifth Session of the International Conference on Sports Biochemistry, sports fatigue was defined as: the physiological process of the organism can not continue its function in the specific level or maintain a predetermined movement. This definition has been recognized by many experts both at home and abroad, which has been widely used. The reason of causing sports fatigue is the movement, after a rest it can be restored. Fatigue is a normal physiological phenomenon, the purpose of study on fatigue is to delay the appearance of fatigue during the motion and try to get recovery as soon as possible after the elimination of fatigue, so as to promote the process of recovery. Sports fatigue can be divided into physical fatigue and mental fatigue, physical fatigue can be reflected by the objective index, which is focused on the subjective feeling of mental fatigue. 


\section{The Parts that Appeared Sports Fatigue}

Any forms of human fatigue are always occurred on a certain part of the body or some parts. In general, the parts that are more prone to have fatigue are the center of nerves moving node plate muscles, etc., according to the different occurrence positions, it can be divided into central fatigue, the fatigue of the nerves muscle's junction and the peripheral fatigue.

\section{Central Fatigue}

During the developing period of sports fatigue, central nervous system can play a dominant role, the occurrence of fatigue is a maintenance inhibition of the central nervous system, which can prevent the excessive body's failure that is occurred in the body.

\section{The Fatigue of the Nerves Muscle's Junction}

The fatigue of the nerves muscle's junction is also called sports central fatigue, sports center is the key part between nerves and muscles, which is the connection and transmission of nerves impulses and muscle contraction, it is also an important part of causing fatigue.

\section{Peripheral Fatigue}

Peripheral fatigue including the changes of all various organs during motion besides nervous system and the muscle's junction.Muscle is the main motion tissue, which can become the research focus and form of the peripheral fatigue.

\section{The Reasons of Causing Sport Fatigue by Playing taekwondo}

The characteristic of taekwondo match such as quick start, abrupt stop and turn, high strength and intense, large load and so on, which belongs to the anaerobic exercise, thus, it must consume a great deal of physical energy.

The fatigue of energy in the body. Some scholars think that the causes of fatigue is due to the large consumption of body's energy substance during having sports, with the enhancement of exercise load, if people can not receive timely supplement, it will cause imbalance by energy supply.

The accumulation of metabolites in the body. The metabolites caused by the movement are largely accumulated in the body, which can not be cleared up in time. These metabolites are including lactic acid, ammonia, carbon dioxide. When people have high intensity exercise, the concentration of lactic acid in the body will raise, which will cause the muscle's fatigue.

The internal environment is disordered. In the fierce competition of the sports item, the muscle will release a large amount of lactic acid and pyruvic acid as well as some other substances, which can be piled up more and more in the body, when people have the external exercise, the catabolic process of human motion is strengthened, the consumption of energy and various nutrients is increased significantly, the hormone effects in the body and the process of enzymatic reaction is very strong, which can emit a lot of water, inorganic salt, electrolyte and lose other trace elements, finally make the body's internal environment changed dramatically, causing the internal environment disordered and occur fatigue. Shown in table 1.

Table 1. The lost amount of inorganic elements in one liter of sweat

\begin{tabular}{cccccccc}
\hline $\begin{array}{c}\text { The } \\
\text { temperature } \\
\text { of dry ball }\end{array}$ & $\begin{array}{c}\text { The } \\
\text { relative } \\
\text { humidity }\end{array}$ & $\mathrm{K}$ & $\mathrm{Na}$ & $\mathrm{Ca}$ & $\mathrm{Mg}$ & $\mathrm{Zn}$ & $\mathrm{Cu}$ \\
$39.3 \pm 1.6$ & $53.1 \pm 6.2$ & $250.6 \pm 11.1$ & $1011.2 \pm 177.4$ & $72.0 \pm 14.9$ & $6.9 \pm 0.8$ & $0.97 \pm 0.26$ & $0.46 \pm 0.14$ \\
\hline
\end{tabular}

The fatigue of central nerves system. When the amount of ATP is reduced during having exercise, amino acid and tea phenol ammonia is increased, the blood glucose is also decreased and people will have other kinds of fatigue. 


\section{The Analysis of the Dietary Intervention of taekwondo Athletes' after the Period of Recovering Fatigue}

\subsection{Drug Therapy}

Using vitamins or natural medicine can effectively regulate the physiological function of human body, accelerate the metabolism, replenish energy, reduce oxygen consumption of tissue, improve blood circulation, provide muscle nutritional supplement. At present, the commonly used drugs for the fatigue recovery of taekwondo are vitamin B1, vitamin B12, vitamin C, Vitamin E, astragalus root, acanthopanax root, ginseng, cordyceps sinensis and pollen, etc.

\subsection{Energy Recovery}

The proper nutrition can make taekwondo athletes keep good physiological function, body composition and exercise abilities, which also can promote the elimination of fatigue, prevent the fatigue induced by exercises. According to the characteristics of taekwondo, adopting nutrition recovery method should pay attention to the following points:

Heat balance: the energy consumption of taekwondo athletes' depends on training and competition intensity, duration, the weight level. taekwondo Athletes should keep the dynamic equilibrium between calorie intake and consumption, so as to maintain weight. According to a survey of Wuhan Institute of physical education with the heat consumption of one hundred and three players, the average is 4318 kilocalorie per kilogram daily, and the daily requirement is about 50 51 kilocalorie per kilogram.

The proportion of pyrogen material should be proper: the energy supply of taekwondo athletes is mainly characterized by muscle glycogen glycolysis and aerobic oxidation of glucose. Therefore, in the diet of taekwondo athletes, the proportion of energy from three major sources:sugar, protein and fat should be followed by the principle: high sugar, high protein, low fat, namely, the proportion of sugar should be $60 \% \sim 70 \%$, the proportion of protein should be $15 \%$ 20\%, while fat should be $10 \% \sim 20 \%$.

Vitamins and minerals should be adequate: when taekwondo athletes take part in the completion, the metabolism is increased, the activity of the enzyme hormone secretion is also increased, at the same time, because of the large number of sweat, the required amount of vitamins and minerals is also increased, therefore, the diet should contain more vitamin $\mathrm{C}$, vitamin $\mathrm{B}$, vitamin $\mathrm{B} 2$, which also should provide sufficient phosphorus and iron. Especially when the athletes have a rest between the games, drinks that are arranged for taekwondo athletes should be rich in minerals and vitamins. Food should be easier to digest, which can be conducive to acid-base balance: taekwondo athletes are often in stressful state with excitement of sympathetic nerve during training and competition, the gastrointestinal blood is less, digestive function is weak. Therefore, they should eat foods that are easily to digest, moreover, they should eat more vegetables, fruits, so as to increase the reservation of body's base.

The dietary system should be reasonable: in addition to breakfast, lunch, dinner, early, it also can increase desert one or two times. Do not drink, do not eat irritating food, paying attention to the hygiene of food. Enough water: because during the movement, people will sweat a lot, body's dehydration is more, therefore, the nutrition should also be added with water. At the same time of adding water, it also should pay attention to add a variety of trace elements, it is better to provide special drinks for taekwondo athletes to drink in the competition and training.

The human body can cause function decline or variation by the extreme fatigue when they have intense training and competition.In order to eliminate fatigue timely and improve body function. The arrangement of diet should pay attention to the following points: do not drink cool drinks too much, such as cold water, fruit juice and so on ; select easily digestible food to eat; eat eggs, sausage and fish that contains animal protein; in order to add salt, drink salt tasty soup or eat salty preserved fruit; eat more fruits, vegetables as the source of vitamin, which can regulate appetite; use milk, milk powder, pig liver and so on to add more vitamins, iron and minerals; add ice cream, chocolate and other desserts; it can be added with some fragrant spicy foods to increase appetite; when the body is tired 
and idle with fatigue with the appetite significantly decreased, the staple food can be changed to eat noodles, porridge and some other foods.

There are many methods to eliminate sports fatigue by Chinese medicine, using a single method is limited, it must be used comprehensively to receive good effect. Because there are a lot of reasons to cause fatigue, and exercising capacities are different individually, so the analysis on fatigue must be considered comprehensively with target. Taekwondo as a sports item, the fatigue is systemic and all rounded. As for the athletes who takes part in continuous games with excessive fatigue can be diagnosed by doctors, they can use the rational drugs to have recovery. After the experiment, the discovery of drugs for taekwondo athlete's recovery had a significant effect. The commonly drugs that are used by taekwondo athletes can be divided into western medicine, Chinese medicine. Now, as for the athletes with excessive fatigue often use injection of ATP. The common Chinese herbal medicine for fatigue recovery is shown in Table 2.

Tab 2. Common Chinese herbal medicine for the recovery of Athletes' fatigue

\begin{tabular}{ccc}
\hline Type of fatigue & Chinese herbal medicine & Effect \\
\hline \multirow{2}{*}{ The nervous system } & Ginseng & \\
& American ginseng & Invigorating Qi, \\
& Salvia miltiorrhiza & Refreshing nerves \\
& Velvet Antler & \\
Chinses Angelica & Rehmannia & Nourishing blood, \\
The cardiovascular system & Red dates & Invigorating blood \\
& Donkey hide gelatin & \\
The muscular system & Ganoderma lucidum & Trichosanthin \\
& Cornus officinalis & Strengthening gluten abd bones, \\
& & Increasing physical power
\end{tabular}

\section{Conclusion}

According to the characteristics of the project in taekwondo sports, the different circumstances of every team member, even the special circumstances of injuries in the team members, a targeted physical training is set up. After a period of targeted rehabilitative physical training, injured area has a good rehabilitation trend. From the test data comparison and the training effect, taekwondo athletes have some effectiveness of proprioception, and the rehabilitation of physical training played a good effect.

\section{Reference}

[1]Avrhama.Y. 2001, Tyrosine improves appetite, cognition and exercise tolenarce in activity Aorexia. Med Sci Sports Exere, Vol.33, p104-110.

[2] Carpenter S, and Karpati G. 1984, Pathology of Skeletal Muscle. New York: Churchill Livingstone, $\mathrm{p}$ 415- 710.

[3]Dioszeghy P and Mechler F. 1988, the significance of simultaneous estimation of serum creatine kinase and myoglobin in neuromus -cular diseases. J Neurol, Vol. 235, p 174-176.

[4]Jayasri D, Narayanans S. 2006, Electro catalytic oxida-tion and amperometric determination of BHA at graphite-wax composite electrode with silver hexacyanoferrate as electrocatalyst. Sensors and Actuators B: Chemical, Vol.119, p 135-142.

[5]Christian Perrin, Liliane Meyer. 2002, Quantification of synthetic phenolic antioxidants in dry foods by reversed-phase HPLC with photodiode array detection. Food Chemistry, Vol.77, p 93-100. 